\title{
A scrutiny on the factors affecting the participation of women doing sports and not doing sports in sportive events
}

\section{Ali Osman KIVRAK ${ }^{1}$, Kadir PEPE ${ }^{2}$, Şirin PEPE $^{3}$, Barbaros Serdar ERDOĞAN ${ }^{2}$}

\author{
${ }^{1}$ Selçuk University, Faculty of Sports Sciences, Konya, Turkey. \\ ${ }^{2}$ Burdur Mehmet Akif Ersoy University, Physical Training and Sports School of Higher Education/Burdur, Turkey \\ ${ }^{3}$ Erenköy Zeki Altındăg Secondary School, Physical Training Teacher/ Konya, Turkey \\ Address Correspondence to AO Kivrak,e-mail: aokivrak@selcuk.edu.tr
}

\begin{abstract}
The study has been conducted in order to scrutinize the factors affecting the participation of women doing sports and not doing sports in sportive events.The population of the study is constituted by Selcuk University, Mehmet Akif Ersoy University, Uludağ University, and Gazi University, with the thought that we can find the individuals from all sections of the Turkish society all together and its sampling group consists of the female students studying at those universities. The data were obtained from written sources and by means of the questionnaire method. A questionnaire oriented to the objective has been developed. The understandability of the questionnaire as well as the validity and reliability of its scope were ensured. The questionnaire's CronbachAlpha reliability coefficient was found as 0,60 . The coefficient is a valid coefficient according to the researchers as well. The questionnaires were filled in through one-to-one discussions in the sampling group by means of the random sampling method. Total 828 women, being 538 doing sports and 290 not doing sports, took part in the study in its state mentioned above. The data obtained were transferred to the computer environment for statistical transaction. As the statistical process, the Chi-Square test processes were conducted for identifying the frequency, \%, Crosstab, and differences. In the determination of the differences, 0,05 was accepted as the level of significance. Upon the assessment of the data obtained, it was determined that the factors affecting women's participation in sportive events are religious reasons, family pressure, government policy, the fact the some branches of sports are not suitable for the physical properties of women, the fact that sportive events inflict damage on their beauties, presence of no suitable areas and venues to perform sports, and the inability of sports culture to take place within the society etc. and that significant differences are present at the 0,05 statistical significance level in the views of the women doing sports and not doing sports in regards to some questions. We can conclude that there are religious, familial, political, physiological, cultural, and spatial factors affecting the participation of women in sportive events.
\end{abstract}

Keywords: Woman, Sportive event, Participation, Factor

\section{INTRODUCTION}

It appears that the technological advancements whose utilization is becoming more and more widespread in every area of the lives of people today have negative impacts on them along with positive ones. Addiction to technology poses negative impacts on humans in terms of physical, social, psychological, and cognitive aspects. Scientists consider sportive events as the only tool that unites people and that has positive impacts in physical, social, psychological, and cognitive grounds.
Sports are the primary basic physical movement of humans within the flow of life in the societies of today. It is known that people take part in sportive events in a regular manner in every location of the world. They have had a lifestyle that intertwined with sports since the very early periods. Hence, it can be said that the existence of sports as a significant social phenomenon is presently an expected outcome. In addition, it appears it is not possible to alienate sports, which have been a social fact presently, from everyday life (5).

We observe that the concept of sports is defined in various ways in line with the viewpoints of the scientists involved with sports, although all 
are essentially the same. Among them, Şahin (16) defines sports as the overall physical, mental, and spiritual activities that aim at the satisfaction of individuals like defeating and becoming capable, that are performed under some particular rules, that are based on competition, and that are socializing and integrating, while the definition of Mirzeoğlu (15) is the physical activities performed in the form of teams or individually in line with predetermined rules, oriented to compete or contest, and attainment of personal perfection.

As can be understood from the definitions, sports and sportive events have some objectives that have physical and social impacts on humans. Among such objectives, the most outstanding ones like the one that is "to create a society that is happy, hardworking, healthy, modern, and dynamic and that has high level of morale and to train generations that know their social duties and responsibilities well, that have proper mental and physical health and morality, and that are creative, productive, constructive, wise, and virtuous, by ensuring that members of families perform sports (18). Sports performed in every section of societies and at any age made people dependent on them directly or indirectly, attracted their attention at all times, and managed to remain vibrant at all times. The fact that sportive activities that bind people to themselves by fulfilling their psychosocial and physiological needs are presently as social institution and harbor symbols regarding people's behaviors, views, beliefs, and thoughts shows that sports have a social structure (12).

People are born to this world in a certain social and geographical environment. The socioeconomic and geographical environment where people are born and grow up causes the emergence of positive or negative impacts on them. Numerous factors affecting people's tendency towards and participation in sportive events that occupy a significant room in their lives are available. Some of such factors are listed by Güner (10) as the individuals' socioeconomic structure, geographical environment, family structure, religious beliefs, social support, and motivation.

The objective of the study has been set to assess the factors and reasons that affect the participation of women in sportive events, which are important in terms of people's physical, social, psychological, and mental development and life quality, in the Turkish society in view of the women doing sports and not doing sports.

The study is important in terms of identifying and analyzing the reasons considered as preventions for the participation of women in sportive events in the Turkish society, ensuring the participation of women in sportive events at utmost levels, as well as creating a society that is happy, hardworking, healthy, modern, and dynamic and that has high level of morale and training generations that know their social duties and responsibilities well, that have proper mental and physical health and morality, and that are creative, productive, constructive, wise, and virtuous, by ensuring the participation in sportive events in every gender and age in the Turkish society.

\section{MATERIAL AND METHOD}

The study has been conducted in order to scrutinize the factors affecting the participation of women doing sports and not doing sports in sportive events.

The population of the study is constituted by Selcuk University, Mehmet Akif Ersoy University, Uludağ University, and Gazi University and its sampling group consists of the female students studying at those universities.

The theoretical data were obtained from written sources and the onsite data were acquired by means of the questionnaire method. A questionnaire oriented to the objective has been developed. The understandability of the questionnaire as well as the validity and reliability of its scope were ensured. The questionnaire's Cronbach Alpha reliability coefficient was found as 0,60 . The coefficient is a valid coefficient according to the researchers as well. The questionnaires were filled in through one-to-one discussions in the sampling group by means of the random sampling method. Total 828 women, being 538 doing sports and 290 not doing sports, took part in the study in its state mentioned above.

The data obtained were transferred to the computer environment for statistical transaction. As the statistical process, the Chi-Square test processes were conducted for identifying the frequency, \%, Crosstab, and differences. In the determination of the differences, 0,05 was accepted as the level of significance. 


\section{FINDINGS}

\begin{tabular}{lcc}
\hline Variables & N & \% Distribution \\
\hline Yes & 538 & 64.1 \\
\hline No & 290 & 35.0 \\
\hline Total & 828 & 100.0 \\
\hline
\end{tabular}

The "states of the participants' doing sports" is questioned in Table 1. Accordingly, it appears that
$64,1 \%$ responded as yes and $35,0 \%$ as no, among the 828 participants taking part in the study.

\begin{tabular}{|c|c|c|c|c|}
\hline Variables & Yes & Partially & No & Total \\
\hline \multirow{2}{*}{ Doing sports } & 94 & 226 & 213 & 533 \\
\hline & $17.6 \%$ & $42.4 \%$ & $40.0 \%$ & $100.0 \%$ \\
\hline \multirow{2}{*}{ Not doing sports } & 44 & 105 & 137 & 286 \\
\hline & $15.4 \%$ & $36.7 \%$ & $47.9 \%$ & $100.0 \%$ \\
\hline \multirow{2}{*}{ Total } & 138 & 331 & 350 & 819 \\
\hline & $16.8 \%$ & $40.4 \%$ & $42.7 \%$ & $100.0 \%$ \\
\hline $\mathrm{X}^{2}=4.796 \quad \mathrm{P}=.091$ & & & & \\
\hline
\end{tabular}

The proposition of "Women do not take part in sportive events due to the fact that women do not attach importance to sports" was questioned in Table 2. It appears in view of aggregate answers of 819 participants answering the question that they were no by $42.7 \%$, partially by $40,4 \%$, and yes by
$16,8 \%$. When the responses are scrutinized in terms of the women doing sports and not doing sports, there is no statistically significant dissension in the responses given between the women doing sports and not doing sports $(\mathrm{P}>0,05)$.

Table 3. The distribution of the responses of the participants to the proposition of "Women do not take part in sportive events due to religious reasons"

\begin{tabular}{|c|c|c|c|c|}
\hline Variables & Yes & Partially & No & Total \\
\hline \multirow{2}{*}{ Doing sports } & 92 & 264 & 182 & 538 \\
\hline & $17.1 \%$ & $49.1 \%$ & $33.8 \%$ & $100.0 \%$ \\
\hline \multirow{2}{*}{ Not doing sports } & 44 & 106 & 140 & 290 \\
\hline & $15.2 \%$ & $36.6 \%$ & $48.3 \%$ & $100.0 \%$ \\
\hline \multirow{2}{*}{ Total } & 136 & 370 & 322 & 828 \\
\hline & $16.4 \%$ & $44.7 \%$ & $38.9 \%$ & $100.0 \%$ \\
\hline $\mathrm{X}^{2}=17.148 \quad \mathrm{P}=.000$ & $\mathrm{P}<0.05$ & & & \\
\hline
\end{tabular}

The proposition of "Women do not take part in sportive events due to religious reasons" was questioned in Table 3. It appears in view of aggregate answers of 828 participants answering the question that they were partially by $44.7 \%$, no by
$38,9 \%$, and yes by $16,4 \%$. When the responses are scrutinized in terms of the women doing sports and not doing sports, there is statistically significant dissension $(\mathrm{P}>0,05)$. 
Table 4. The distribution of the responses of the participants to the proposition of "Women do not take part in sportive events due to family pressure"

\begin{tabular}{|c|c|c|c|c|}
\hline Variables & Yes & Partially & No & Total \\
\hline \multirow{2}{*}{ Doing sports } & 116 & 241 & 179 & 536 \\
\hline & $21.0 \%$ & $45.0 \%$ & $33.4 \%$ & $100.0 \%$ \\
\hline \multirow{2}{*}{ Not doing sports } & 48 & 115 & 128 & 291 \\
\hline & $16.0 \%$ & $39.5 \%$ & $44.0 \%$ & $100 . \%$ \\
\hline \multirow{2}{*}{ Total } & 164 & 356 & 307 & 827 \\
\hline & $19.8 \%$ & $43.0 \%$ & $37.1 \%$ & $100.0 \%$ \\
\hline$X^{2}=9.517$ & $\mathrm{P}<0.05$ & & & \\
\hline
\end{tabular}

The distribution of the responses of the participants to proposition of "Women do not take part in sportive events due to family pressure" is present in Table 4. It appears in view of the aggregate answers of the 827 participants responding to this question that they were partially by $43,0 \%$, no by $37,1 \%$, and yes by $19,8 \%$. When it comes to the responses of the participants doing sports and not doing sports, 45,0 $\%$ of the ones doing sports responded as partially while $44,0 \%$ the ones not doing sports responded as no. This result shows that there is statistically significant dissension $(\mathrm{P}<0,05)$.

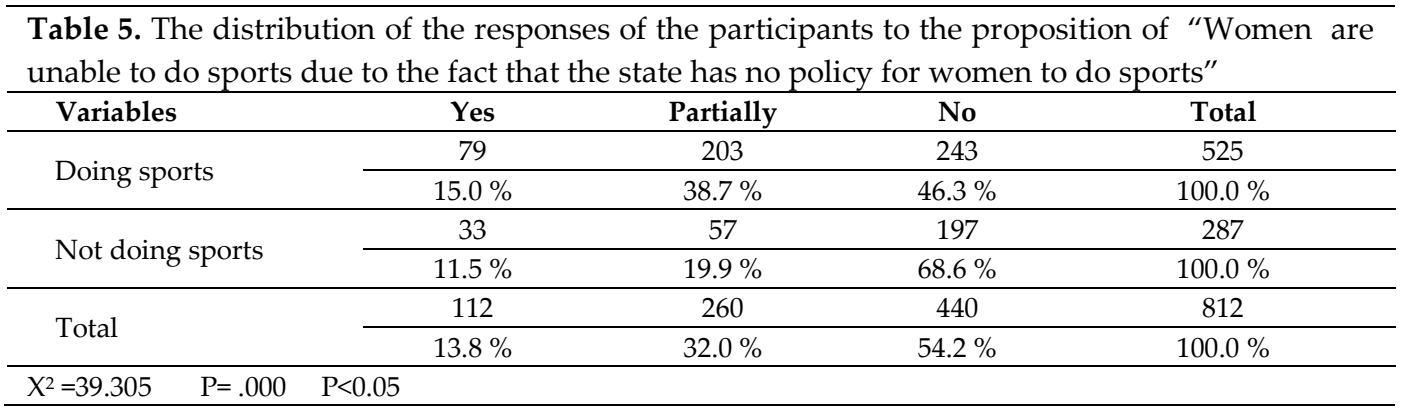

The proposition of "Women are unable to do sports due to the fact that the state has no policy for women to do sports" was questioned in Table 5. It appears in view of aggregate answers of 812 participants answering the question that they were no by $54.2 \%$, partially by $32,0 \%$, and yes by $13,8 \%$.
When it comes to the responses of the participants doing sports and not doing sports, $46,3 \%$ of the ones doing sports responded as yes while $68,6 \%$ the ones not doing sports responded as yes. We can say according to those responses that there is a statistically significant dissension $(\mathrm{P}>0,05)$.

Table 6. The distribution of the responses of the participants to the proposition of "They are unable to take part in sportive events due to the fact that some branches of sports are not suitable for women's physical and physiological structures"

\begin{tabular}{|c|c|c|c|c|}
\hline Variables & Yes & Partially & No & Total \\
\hline \multirow{2}{*}{ Doing sports } & 159 & 272 & 101 & 532 \\
\hline & $29.9 \%$ & $51.1 \%$ & $19.0 \%$ & $100.0 \%$ \\
\hline \multirow{2}{*}{ Not doing sports } & 89 & 133 & 65 & 287 \\
\hline & $31.0 \%$ & $46.3 \%$ & $22.6 \%$ & $100.0 \%$ \\
\hline \multirow{2}{*}{ Total } & 248 & 405 & 166 & 819 \\
\hline & $30.3 \%$ & $49.5 \%$ & $20.3 \%$ & $100.0 \%$ \\
\hline
\end{tabular}

The proposition of "They are unable to take part in sportive events due to the fact that some branches of sports are not suitable for women's physical and physiological structures" was questioned in Table 6.
It appears in view of aggregate answers of 819 participants answering the question that they partially by $49,5 \%$, yes by $30,3 \%$, and no by $20,3 \%$. When it comes to the responses of the participants doing sports and not doing sports, there is a 
statistically significant dissension in the answers given $(P>0,05)$.

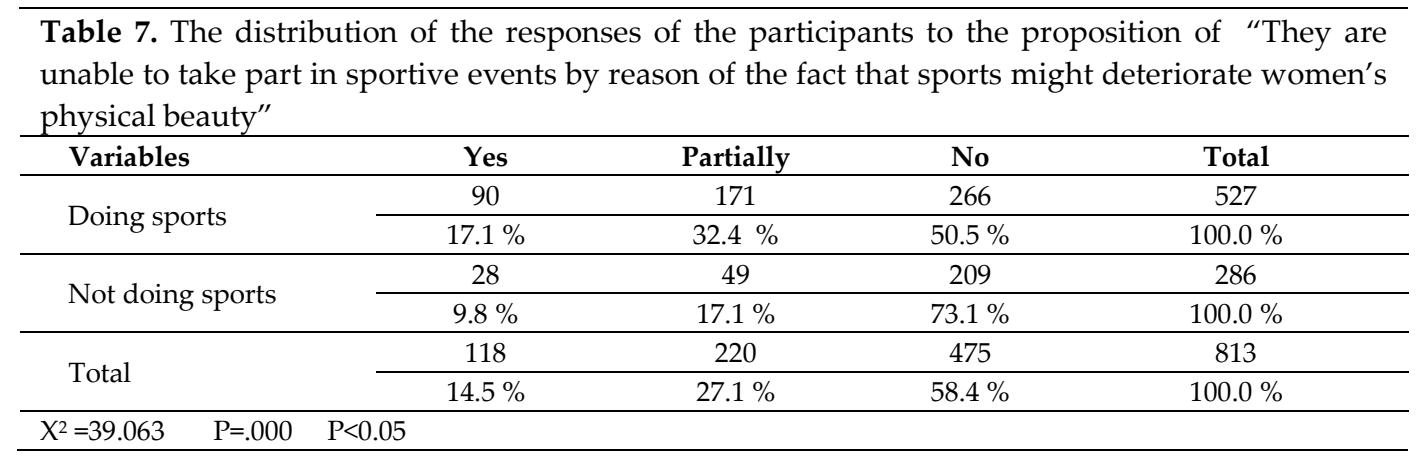

The proposition of "They are unable to take part in sportive events by reason of the fact that sports might deteriorate women's physical beauty" was questioned in Table 7. It appears in view of the aggregate answers of the 813 participants responding to this question that they were no by $58.4 \%$, partially by $27,1 \%$, and yes by $14,5 \%$.
When it comes to the responses of the participants doing sports and not doing sports, 50,5\% of the ones doing sports responded as no and $32,4 \%$ as partially, while $73,1 \%$ the ones not doing sports responded as no and $17,1 \%$ as partially. This result is statistically significant $(\mathrm{P}<0,05)$.

Table 8. The distribution of the responses of the participants to the proposition of "Many women are unable to take part in sportive events due to their lack of time for doing sports"

\begin{tabular}{|c|c|c|c|c|}
\hline Variables & Yes & Partially & No & Total \\
\hline \multirow{2}{*}{ Doing sports } & 190 & 260 & 87 & 537 \\
\hline & $35.4 \%$ & $48.4 \%$ & $16.2 \%$ & $100.0 \%$ \\
\hline \multirow{2}{*}{ Not doing sports } & 128 & 116 & 44 & 288 \\
\hline & $44.4 \%$ & $40.3 \%$ & $15.3 \%$ & $100.0 \%$ \\
\hline \multirow{2}{*}{ Total } & 318 & 376 & 131 & 825 \\
\hline & $38.5 \%$ & $45.6 \%$ & $15.9 \%$ & $100.0 \%$ \\
\hline
\end{tabular}

The proposition of "Many women are unable to take part in sportive events due to their lack of time for doing sports" was questioned in Table 8. It appears in view of aggregate answers of 825 participants answering the question that they were partially by
$45.6 \%$, yes by $38,5 \%$, and no by $15,9 \%$. When the responses of the participants are analyzed in terms of doing sports and not doing sports, there seems to be no statistically significant dissension in the answers given $(\mathrm{P}>0,05)$.

Table 9. The distribution of the responses of the participants to the proposition of "Women cannot take part in sportive events due to the fact that women are alienated in the field of sports as in many areas in our society"

\begin{tabular}{|c|c|c|c|c|}
\hline Variables & Yes & Partially & No & Total \\
\hline \multirow{2}{*}{ Doing sports } & 176 & 266 & 91 & 533 \\
\hline & $33.0 \%$ & $49.9 \%$ & $17.1 \%$ & $100.0 \%$ \\
\hline \multirow{2}{*}{ Not doing sports } & 115 & 123 & 53 & 291 \\
\hline & $39.5 \%$ & $42.3 \%$ & $18.2 \%$ & $100.0 \%$ \\
\hline \multirow{2}{*}{ Total } & 291 & 389 & 144 & 824 \\
\hline & $35.3 \%$ & $47.2 \%$ & $17.5 \%$ & $100.0 \%$ \\
\hline $\mathrm{X}^{2}=4.717 \quad \mathrm{P}=.095 \quad \mathrm{P}>0.05$ & & & & \\
\hline
\end{tabular}

The proposition of "Women cannot take part in sportive events due to the fact that women are alienated in the field of sports as in many areas in our society" was questioned in Table 9. It appears in view of aggregate answers of 824 participants answering the question that they were partially by
$47.2 \%$, yes by $35,3 \%$, and no by $17,5 \%$. When the responses of the participants are analyzed in terms of doing sports and not doing sports, there seems to be no statistically significant dissension in the answers given $(\mathrm{P}>0,05)$. 
Table 10. The distribution of the responses of the participants to the proposition of "Women cannot take part in sportive events due to the fact that there are no areas and venues to do sports"

\begin{tabular}{|c|c|c|c|c|}
\hline Variables & Yes & Partially & No & Total \\
\hline \multirow{2}{*}{ Doing sports } & 230 & 241 & 65 & 536 \\
\hline & $42.9 \%$ & $45.0 \%$ & $12.1 \%$ & $100.0 \%$ \\
\hline \multirow{2}{*}{ Not doing sports } & 127 & 120 & 42 & 289 \\
\hline & $43.9 \%$ & $41.5 \%$ & $14.5 \%$ & $100.0 \%$ \\
\hline \multirow{2}{*}{ Total } & 357 & 361 & 107 & 825 \\
\hline & $43.3 \%$ & $43.8 \%$ & $13.0 \%$ & $100.0 \%$ \\
\hline
\end{tabular}

The proposition of "Women cannot take part in sportive events due to the fact that there are no areas and venues to do sports" was questioned in Table 10. It appears in view of aggregate answers of 825 participants answering the question that they were partially by $43.8 \%$, yes by $43,3 \%$, and no by $13.0 \%$. When the responses of the participants doing sports and not doing sports are analyzed, there seems to be no statistically significant dissension in the answers given $(\mathrm{P}>0,05)$.

\begin{tabular}{|c|c|c|c|c|}
\hline Variables & Yes & Partially & No & Total \\
\hline \multirow{2}{*}{ Doing sports } & 206 & 252 & 74 & 532 \\
\hline & $38.7 \%$ & $47.4 \%$ & $13.9 \%$ & $100.0 \%$ \\
\hline \multirow{2}{*}{ Not doing sports } & 133 & 117 & 40 & 290 \\
\hline & $45.9 \%$ & $40.3 \%$ & $13.8 \%$ & $100.0 \%$ \\
\hline \multirow{2}{*}{ Total } & 339 & 369 & 114 & 822 \\
\hline & $41.2 \%$ & $44.9 \%$ & $13.9 \%$ & $100.0 \%$ \\
\hline
\end{tabular}

The proposition of "Women are unable to take part in sportive events due to the failure of creating a sports culture in the society" was questioned in Table 11. It appears in view of aggregate answers of 822 participants answering the question that they were partially by $44.9 \%$, yes by $41,2 \%$, and no by $13,9 \%$. When the responses of the participants doing sports and not doing sports are analyzed, there is no statistically significant dissension in the answers given $(\mathrm{P}>0,05)$.

\section{DISCUSSION AND RESULT}

The study has been conducted in order to scrutinize the factors affecting the participation of women doing sports and not doing sports in sportive events. 828 female participants took part in the study. $64,1 \%$ of them do sports and $35,0 \%$ do not do sports (Table 1 ).

The responses of the participating women doing and not doing sports for the questions regarding their participation in sportive events were statistically assessed and discussed.
The proposition of "Women do not take part in sportive events due to the fact that women do not attach importance to sports in the Turkish society" was responded by 819 participants. It appears in view of their aggregate answers that they were no by $42,7 \%$, partially by $40,4 \%$, and yes by $16,8 \%$. The comparative $\mathrm{X}^{2}$ analysis value of their responses was found as 4,796 (Table 2). This value is not significant at the 0,05 significance level $(P>0,05)$. That is to say, there is no significant dissensus in the responses provided for the questions for the women doing sports and not doing sports. According the foregoing values obtained, we can say that the idea that women do not take part in sportive events due to the fact that women do not attach importance to sports in the Turkish society is not an effective view. That is to say, the idea that for the women doing sports and not doing sports, they do not attach importance to sports does not affect the participation in sportive events.

The proposition of "Women do not take part in sportive events due to religious reasons" was 
responded by 828 participants. It appears in view of their aggregate answers that they were partially by $44,7 \%$, no by $38,9 \%$, and yes by $16,4 \%$. When it comes to the responses of the participants doing sports and not doing sports, $49,1 \%$ of the ones doing sports responded as partially and $33,8 \%$ as no while $36,6 \%$ the ones not doing sports responded as partially and $48,3 \%$ as no. The comparative $X^{2}$ analysis value of their responses was found as 17,148 (Table 3). This value is significant at the 0,05 significance level $(\mathrm{P}<0,05)$. That is to say, there is dissensus in the responses provided for the questions regarding the fact that participation of women doing sports and not doing sports in sportive events is affected by religious reasons. According the foregoing values, we can say as compared with women not doing sports that the women doing sports think that the participation of women in sportive events is affected by religious reasons. The findings of the study conducted by Arab-Moghaddam et al. (1) that the level of participation of Muslim women in leisure and sportive events is low as well as the view of Fasting and Pfister (7) and Grant (8) that religious views have significant share in the fact that the women are almost second to none in the sportive institutions and participation in sports in Islamic countries are supportive of our finding.

The proposition of "Women do not take part in sportive events due to family pressure" was responded by 827 participants. It appears in view of their aggregate answers that they were partially by $43,0 \%$, no by $37,1 \%$, and yes by $19,8 \%$. When it comes to the responses of the participants doing sports and not doing sports, $45,0 \%$ of the ones doing sports responded as partially while $44,0 \%$ of the ones not doing sports responded as no. The comparative $X^{2}$ analysis value of their responses was found as 9,517 (Table 4). This value is statistically significant at the 0,05 significance level $(\mathrm{P}<0,05)$. That is to say, there is dissensus between the ones doing sports and not doing sports. According the foregoing values obtained, we can say as compared with women not doing sports that the women doing sports think that family pressure is effective in women's participation in sports. The view in the researches of McIntyre and Rhodes (14) that the support provided particularly by families to women affects the participation in leisure and sportive events as well as the finding of Biddle et al. (3) on a study on young women regarding the fact that family support has significant role in the tendency toward sports are in parallel with the finding of our study.

The proposition of "Women are unable to do sports due to the fact that the state has no policy for women to do sports" was responded by 812 participants. It appears in view of their aggregate answers that they were no by $54,2 \%$, partially by $32,0 \%$, and yes by $13,8 \%$. When it comes to the responses of the participants doing sports and not doing sports, $46,3 \%$ of the ones doing sports responded as yes while $68,6 \%$ the ones not doing sports responded as yes. The comparative $\mathrm{X}^{2}$ analysis value of their responses was found as 39,305 (Table 5). This value shows that a significant dissensus is present $(\mathrm{P}<0,05)$. According the foregoing data, we can say as compared with women doing sports that the women not doing sports think that lack of a state policy for women to do sports is effective in women's participation in sports.

The proposition of "They are unable to take part in sportive events due to the fact that some branches of sports are not suitable for women's physical and physiological structures" was responded by 819 participants. It appears in view of their aggregate answers that they were partially by $49,5 \%$, yes by $30,3 \%$, and no by $20,3 \%$. The comparative $X^{2}$ analysis value of the responses of the participants doing sports and not doing sports was found as 2,176 (Table 6). This value is not statistically significant at the 0,05 significance level $(\mathrm{P}<0,05)$ That is to say, there is no dissensus between the ones doing sports and not doing sports. Upon the foregoing data obtained, we can say that according to the participants doing sports and not doing sports, women do not take part in sportive events due to the fact that some branches of sports are not suitable for women's physical and physiological structures.

The proposition of "They are unable to take part in sportive events by reason of the fact that sports might deteriorate their physical beauty" was responded by 813 participants. It appears in view of their aggregate answers that they were no by $58.4 \%$, partially by $27,1 \%$, and yes by $14,5 \%$. When it comes to the responses of the participants doing sports and not doing sports, 50,5\% of the ones doing sports responded as no and $32,4 \%$ partially, while $73,1 \%$ of the ones not doing sports responded as no 
and $17,1 \%$ as partially. The comparative $\mathrm{X}^{2}$ analysis value of the responses of the ones doing sports and not doing sports was found as 39,063 (Table 7). This value shows that a significant dissensus is present $(P<0,05)$. According to the values obtained, we can say that the participants not doing sports are in more disagreement than the ones doing sports that women are unable to take part in sportive events by reason of the fact that sports might deteriorate their physical beauty. That is to say, we can consider in view of the results obtained, the reason of deterioration of the beauty of women is not an efficient factor in terms of not participating in sportive events. When the research findings taking place in the literature is assessed, it appears that the prime factor steering women toward sports and leisure events is the wish of weight loss (Aytan) (2) and having a good physical appearance $(13,17)$. These findings are supportive of our finding.

The proposition of "Many women are unable to take part in sportive events due to their lack of time for doing sports" was responded by 825 participants. It appears in view of their aggregate answers that they were partially by $45.6 \%$, yes by $38,5 \%$, and no by $15,9 \%$. The comparative $X^{2}$ analysis value of the responses of the ones doing sports and not doing sports was found as 6,820 (Table 8). This value is not significant at the 0,05 significance level $(\mathrm{P}>0,05)$. That is to say, there is no dissensus between the ones doing sports and not doing sports. According to the values obtained, we can say that women are unable to take part in sportive events due to their lack of time for doing sports in general. Currie (4) has found that women, who are mothers, cannot take time for leisure and sportive events and Kraus (11) conducts studies for increasing the life quality of the families of the women who are married and have a profession. Hence, women cannot take time to participate in sportive events. Their views are supportive of our finding.

The proposition of "Women cannot take part in sportive events due to the fact that women are alienated in the field of sports as in many areas in our society" was responded by 824 participants. It appears in view of their aggregate answers that they were partially by $47.2 \%$, yes by $35,3 \%$, and no by $17,5 \%$. The comparative statistical $X^{2}$ analysis value

In the study conducted by Fasting and Pfister (7) in terms of women's participation in of the responses of the ones doing sports and not doing sports was found as 4,717 (Table 9). This value is not significant at the 0,05 significance level $(\mathrm{P}>0,05)$. According to the values obtained, we can say that the participants consider that the reason for women to be unable to take part in sportive events is due to the fact that women are alienated in the field of sports as in many areas in our society. Eitzen and Sage (6) think that particularly modern sports have developed as the most important indicator of the strength and superiority of men and Giulianotti's (9) view is that the impact of the powerful paternalistic understanding in societies alienate women from sportive events and has a negative aspect. This view tallies with the finding of the study.

The proposition of "Women cannot take part in sportive events due to the fact that there are no areas and venues to do sports" was responded by 825 participants. It appears in view of their aggregate answers that they were partially by 43.8 $\%$, yes by $43,3 \%$, and no by $13 \%$. The comparative statistical $\mathrm{X}^{2}$ analysis value of the responses of the ones doing sports and not doing sports was found as 1,392 (Table 10). This value is not significant at the 0,05 significance level $(P>0,05)$. According to the values obtained, we can say that participants mainly think that presence of no areas and venues to do sports are effective in women's failure to take part in sportive events.

The proposition of "Women are unable to take part in sportive events due to the failure of creating a sports culture in the society" was questioned. It appears in view of aggregate answers of 822 participants answering the question that they were partially by $44.9 \%$, yes by $41,2 \%$, and no by $13,9 \%$. The comparative statistical $X^{2}$ analysis value of the responses of the ones doing sports and not doing sports was found as 4,385 (Table 11). This value shows that there is no dissensus at the 0,05 significance level $(\mathrm{P}>0,05)$. That is to say, there is no dissensus in terms of the view of "Women are unable to take part in sportive events due to the failure of creating a sports culture in the society" among the participant women doing sports and not doing sports. According to the values obtained, we can say participants mainly think that women are unable to take part in sportive events due to the failure of creating a sports culture in the society.

sports in Turkey, they state that the factors like the social status and place of residence, family relations, 
importance of the success at school, consideration that sports are male-specific activities, aggravated conditions of working, importance of family, the fact that women do not consider sports as entertainment, body and beauty perception, and covering, affect women's participation in sports negatively. Such findings and views generally tally with the findings of our study.

As a result of the research findings obtained, we can say that the participation of women in sportive events in the Turkish society are affected by the factors like religious beliefs, family pressure, the fact that the state has no policy for women to do sports, the fact that some branches of sports are not suitable for women's physical and physiological structures, women's inability to take time for sportive exercises, alienation of women from participation in sportive exercises, insufficiency of place and venue for sportive exercises, and failure of the existence of a sports culture in the society.

\section{Suggestions:}

- A sports policy that will ensure women's participation in sports must be developed

- The fact that the religious beliefs are not prevention for participation in sports must be explained to public

- Areas and venues where women will take part in sportive exercises must be prepared

- A sports culture must be created within the society

- Awareness must be raised among families in terms of exercises to be done by women

- Benefits of sports must be made known at the educational institutions and students must be provided with the habit of performing sportive exercises

- Alienation of women from sportive activities in the society must be prevented

- Women's participation in sports must be encouraged

- The reasons that prevent women from participating in sports must be researched from time to time and the preventions identified must be eliminated

\section{REFERENCES}

1. Arab-Moghaddam N, Henderson KA, Sheikholeslami R. Women's leisure and constraints to participation: Iranian perspectives. Journal of Leisure Research, 2007; 39(1): 109-126.

2.Aytan GK. Kadınların spora olan ilgilerininin İcelenmesi.Kastamonu Eğitim Dergisi, 2013; 21(2): 777790.

3. Biddle SJ, Whitehead SH, O Donovan TM, Nevill ME. Correlates ofparticipation in physical activity for adolescent girls: A systematic review of recent literature. Journal of Physical Activity\& Health, 2005; 2(4): 423-434.

4. Currie J. Motherhood, stress and the exercise experience: Freedomor constraint, Leisure Studies, 2004; 23(3): 225-242.

5. Dever A. Sporsosyolojisi, BaşlıkYayınları, İstanbul, 2010.

6. Eitzen DS, Sage GH. Sociology of North American Sport, SixthEdition, McGraw-Hill, Boston, 1997.

7. Fasting K, Pfister G. Opportunities and Barriers for Women inSport: Turkey. Women of Diversity Productions Inc., Las Wegas, 1999.

8. Grant J. Sport, Culture and Society: An Introduction. Routledge, Oxon, 2006.

9. Giulianotti R Sport, A Critical Sociology. Polity Press, Cambridge, 2005.

10.Güner B. Kadınların spor ve serbest zaman etkinliklerine katılım sorunlarının değerlendirilmesi. Uluslararası Spor Bilimleri Dergisi, 2015; 1(1).

11.Kraus RG. Recreation Today: Program Planning and Leadership. 2. Edition. Goodyear Publisling.California, 1997.

12.Küçük V, Koç H. Psiko-Sosyal gelişim süreci içerisinde insan ve spor ilişkisi, Dumlupınar Üniversitesi Sosyal Bilimler Dergisi, 2004; 10: 131-141.

13.Liechty T, Freeman PA, Zabriskie RB. Body İmage and Beliefs About Appearance: Constraints on The Leisure of CollegeAge and Middle-Age Women. Leisure Sciences, 2006; 28(4): 311-330.

14.Mclntyre CA, Rhodes RE. Correlates Of Leisure-Time Physical Activity During Transitions To Motherhood. Women $\mathcal{E}$ Health, 2009; 49(1): 66-83.

15.Mirzeoğlu N. Sporun Bilimsel Temelleri, Spor Bilimlerine Giriş, ed: Mirzeoğlu N, BağırganYayınevi, pp: 85, Ankara, 2003.

16.Şahin HM. Beden Eğitimi ve Sporda Temel Kavramlar, Nobel Yayın Dağıtım, pp: 370,

Gaziantep, 2002.

17.Sit CH, Kerr JH, Wong IT. Motives For and Barriers To Physical Activity Participation in iddle-Aged Chinese Women.Psychology of Sport and Exercise, 2008; 9(3): 266- 283.

18. Yetim AA. Sosyoloji ve Spor, Morpa Kültür Yayınları, İstanbul,2005. 